\title{
The Analysis of the Rotating Disk of some Southern Galactic B[e] Stars
}

\author{
Marcelo Borges Fernandes, H.J.G.L.M. Lamers, Michaela Kraus \\ Astronomical Institute, Utrecht University, P.O. Box 80000, NL-3508, \\ TA, Utrecht, The Netherlands \\ Francisco X. de Araújo \\ Observatório Nacional, Rua General José Cristino, 77, CEP: \\ 20921-400, São Cristóvão, Rio de Janeiro, Brasil
}

\begin{abstract}
The spectra of stars with the $\mathrm{B}[\mathrm{e}]$ phenomenon are dominated by features that are related to physical conditions of circumstellar material around these objects and are not intrinsic to the stars. Previous studies have shown that emission lines present in the optical spectra of these objects are formed in an equatorial rotating disk. The analysis of high and low resolution spectra, obtained by us with 1.52 telescope in ESO for some Southern Galactic B[e] Stars, can give us information about the structure and velocity of the disk. We will describe the analysis of the unclassified B[e] star Hen 2-90.
\end{abstract}

\section{Introduction}

From a sample of stars with the B[e] phenomenon (Lamers et al. 1998) observed by us with the FEROS and B\&C spectrograph at 1.52 telescope in ESO (La Silla, Chile - agreement ESO/ON), we have analyzed the circumstellar medium around Hen 2-90, a unclassified B[e] star (that is sometimes classified in the literature as a compact planetary nebulae) considering the presence of a rotating disk. Its presence is suggested by images taken with WFPC2 in HST (Sahai \& Nyman 2000) and also by the presence of double peaked profiles in its spectrum. Our analysis is based on the comparison between the observed luminosity of [S II] lines and $\mathrm{H} \alpha$ with those predicted by a model that will be described below.

\section{Double Peaks}

The existence of a rotating disk at Hen 2-90 is confirmed by the presence of many forbidden and permitted lines with double peaks (see Figure 1). From the separation between two peaks we have found a $v$ sin $i \sim 20 \mathrm{~km} \mathrm{~s}^{-1}$. 


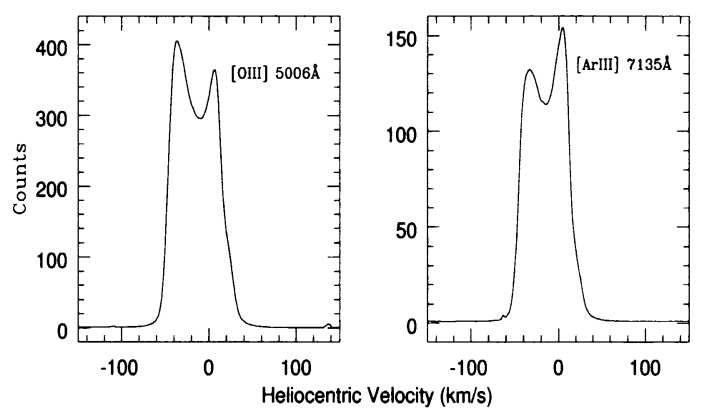

Figure 1 -[O III] and [ArIII] double peaked profiles present in the high resolution spectra (FEROS) of Hen 2-90.

\section{Wind plus a Disk: Our Model}

- 2 spherical envelopes, considering one representing the polar wind and the other representing the disk. The ionization is calculated with a photoionization model for $\mathrm{H}, \mathrm{He}$ and $\mathrm{S}$. One envelope has a higher density than the other and we only use the equatorial sector with a width of 15 degrees. The other has a lower density and we are considering the luminosity that comes from the remaining region, outside the disk.

- $\dot{M}$ is an input parameter

- The [S II] lines come only from the disk and $\mathrm{H} \alpha$ has two components that are coming from both regions.

\section{Results and Conclusions}

We have found a $\dot{M} \sim 10^{-9} \mathrm{M}_{\odot} \mathrm{yr}^{-1}$ for the disk region, if all $\mathrm{S}$ is $\mathrm{S}$ II. This value is low compared with other compact planetary nebula. If the S II fraction (S II/S) is only $10^{-3}$, then the $\dot{M}$ is $\sim 10^{-7} \mathrm{M}_{\odot} \mathrm{yr}^{-1}$, because we have found that $\dot{M}$ is proportional to $(\mathrm{S} \mathrm{II} / \mathrm{S})^{-0.67}$ (Borges Fernandes et al. 2003).

We will apply this model for other stars in our sample. For this, we will improve the model including other elements like $\mathrm{O}$ and $\mathrm{N}$.

\section{References}

Borges Fernandes, M., Lamers, H.J.G.L.M., Kraus, M. \& de Araújo, F.X. 2003, in preparation

Lamers, H.J.G.L.M., Zickgraf, F.-J., de Winter, D., Houziaux, L. \& Zorec, J. 1998, A\&A 340,117

Sahai, R., \& Nyman, L.-A. 2000, ApJ 537, L145 